\title{
Prediction Model for Freedom from TLR from a Multi-study Analysis of Long-Term Results with the Zilver PTX Drug-Eluting Peripheral Stent
}

\author{
Michael D. Dake ${ }^{1} \cdot$ Fabrizio Fanelli $^{2} \cdot$ Aaron E. Lottes $^{3} \cdot$ Erin E. O'Leary $^{4}$. \\ Heidi Reichert ${ }^{5} \cdot$ Xiaohui Jiang ${ }^{5}$. Weiguo $\mathrm{Fu}^{6}$ - Osamu Iida ${ }^{7} \cdot \mathrm{Kan}^{6} \mathrm{Zen}^{8}$. \\ Marc Schermerhorn ${ }^{9}$ Thomas Zeller ${ }^{10}$ - Gary M. Ansel ${ }^{11}$
}

Received: 4 August 2020/Accepted: 8 September 2020/Published online: 6 October 2020

(C) The Author(s) 2020

\begin{abstract}
Purpose Develop a prediction model to determine the impact of patient and lesion factors on freedom from target lesion revascularization (ffTLR) for patients who are candidates for Zilver PTX drug-eluting stent (DES) treatment for femoropopliteal lesions.

Methods Patient factors, lesion characteristics, and TLR results from five global studies were utilized for model development. Factors potentially associated with TLR (sex, age, diabetes, hypertension, hypercholesterolemia, renal disease, smoking status, Rutherford classification, lesion length, reference vessel diameter (RVD), popliteal involvement, total occlusion, calcification severity, prior interventions, and number of runoff vessels) were analyzed in a Cox proportional hazards model. Probability of ffTLR
\end{abstract}

Electronic supplementary material The online version of this article (https://doi.org/10.1007/s00270-020-02648-6) contains supplementary material, which is available to authorized users.

Michael D. Dake

mddake@email.arizona.edu

1 The University of Arizona, Health Sciences Innovation Building, 1670 East Drachman Street, 9th Floor SVP Suite, P.O. Box 210216, Tucson, AZ 85721-0216, USA

2 Department of Vascular and Interventional Radiology, "Careggi" University Hospital, Florence, Italy

3 Purdue University, West Lafayette, IN, USA

4 Cook Research Incorporated, West Lafayette, IN, USA

5 EpidStrategies, Ann Arbor, MI, USA

6 Department of Vascular Surgery, Zhongshan Hospital, Fudan University, Shanghai, China

7 Cardiovascular Center, Kansai Rosai Hospital, Amagasaki, Japan was generated for three example patient profiles via combinations of patient and lesion factors. TLR was defined as reintervention performed for $\geq 50 \%$ diameter stenosis after recurrent clinical symptoms.

Results The model used records from 2227 patients. The median follow-up time was 23.9 months (range: 0.03-60.8). The Kaplan-Meier estimates for ffTLR were $90.5 \%$ through 1 year and $75.2 \%$ through 5 years. In a multivariate analysis, sex, age, Rutherford classification, lesion length, RVD, total occlusion, and prior interventions were significant factors. The example patient profiles have predicted 1-year ffTLRs of 97.4, 92.3, and $86.0 \%$ and 5 -year predicted ffTLRs of 92.8, 79.5, and 64.8\%. The prediction model is available as an interactive web-based tool (https://cooksfa.z13.web.core.windows.net).

Conclusions This is the first prediction model that uses an extensive dataset to determine the impact of patient and lesion factors on ffTLR through 5 years and provides an

8 Department of Cardiovascular Medicine, Graduate School of Medical Science, Kyoto Prefectural University of Medicine, Kyoto, Japan

9 Division of Vascular Surgery, Department of Surgery, Beth Israel Deaconess Medical Center, Boston, MA, USA

10 Universitaets-Herz-Zentrum Freiburg - Bad Krozingen, Bad Krozingen, Germany

11 Department of Medicine, Ohio Health/Riverside Methodist Hospital, Columbus, OH, USA 
interactive web-based tool for expected patient outcomes with the Zilver PTX DES.

Clinical Trial Registrations Zilver PTX RCT unique identifier: NCT00120406; Zilver PTX single-arm study unique identifier: NCT01094678; Zilver PTX China study unique identifier: NCT02171962; Zilver PTX US post-approval study unique identifier: NCT01901289; Zilver PTX Japan post-market surveillance study unique identifier: NCT02254837.

Levels of Evidence Zilver PTX RCT: Level 2, randomized controlled trial; Single-arm study: Level 4, large case series; China study: Level 4, case series; US post-approval study: Level 4, case series Japan PMS study: Level 4, large case series.

Keywords Drug-eluting stent · Paclitaxel ·

Peripheral artery disease $\cdot$ Prediction model $\cdot$ Target lesion revascularization

\section{Introduction}

The management of symptomatic peripheral artery disease (PAD) is frequently a complex challenge influenced by a variety of patient factors and anatomic characteristics of the disease. In an effort to reduce restenosis, the most common cause of failure, following endovascular intervention, drug-eluting stents (DES) were developed $[1,2]$ with the hope of providing a safe and durable endovascular option for treatment of patients with PAD.

Endovascular drug-based therapies for PAD have consistently shown superior patency and freedom from target lesion revascularization (ffTLR) outcomes relative to traditional devices [e.g., standard percutaneous balloon angioplasty (PTA) and bare metal stents (BMS)] [3, 4]. These drug-based therapies have demonstrated long-term effectiveness [5, 6].

Despite improved results with drug-based technologies, a limitation of these endovascular devices is still restenosis. Patient-level data of coronary DES have been pooled to analyze which factors are predictors for revascularization [7]. In terms of risk factors for revascularization after femoropopliteal DES therapy, including patient demographic and clinical variables, limited data exist. The purpose of this study is to develop a prediction model using patient-level data from five prospective clinical trials to determine the impact of patient and lesion factors on ffTLR for patients who are candidates for DES treatment.

\section{Methods}

The global clinical program for the DES (Zilver PTX, Cook Medical, Bloomington, IN, USA) consists of multiple Cook-sponsored pre-market (i.e., Zilver PTX randomized controlled trial [RCT], single-arm study [SAS], and China study) and post-market clinical (i.e., US post-approval study [US PAS] and Japan post-market surveillance study [PMS]) studies. A detailed description of the study design, inclusion and exclusion criteria, and results have been previously published for three of the five trials included in this analysis [1, 5, 8-11]. Table 1 describes the study characteristics for the five studies included in the current analysis.

Data from the five global studies of the DES were combined for the post hoc analysis based on factors that were defined consistently across and the availability of these factors to address differential patient risk for TLR across all studies. Common patient-level characteristics hypothesized to be associated with TLR and utilized for model development included sex, age $(<65,65-74$, $75-84, \geq 85$ years), diabetes, hypertension, hypercholesterolemia, renal disease (e.g., hematuria, chronic urinary tract infections, renal calculi, renal insufficiency), smoking history (never, past, current), Rutherford classification (claudicant, critical limb ischemia [CLI]), lesion length ( $50 \mathrm{~mm}$ buckets, from $<50 \mathrm{~mm}$ to $\geq 300 \mathrm{~mm}$ ), reference vessel diameter $(<5 \mathrm{~mm}$ vs. $\geq 5 \mathrm{~mm}$ ), popliteal involvement, total occlusion, calcification severity (none, mild/moderate, severe), and prior intervention of the study lesion. When available, core laboratory data were used. The continuous patient-level characteristics (i.e., age, Rutherford classification, lesion length, and reference vessel diameter) were categorized in order to facilitate the prediction model and limit the number of combinations. TLR was defined in all studies as reintervention performed for $\geq 50 \%$ diameter stenosis after recurrent clinical symptoms. As pre-specified, 5-year follow-up was defined as the window of 4.5 years through the end of the followup window.

Baseline characteristics were summarized using frequencies and percentages. A Kaplan-Meier analysis was performed to assess overall freedom from TLR. Cox proportional hazards models were fit to predict freedom from TLR using both a univariate and multivariate approach. Validation was performed using a 60/40 training/test split of the dataset to evaluate model discrimination and calibration [12]. A 60/40 split was chosen in order to provide sufficient observations in each set, while preventing possible overfitting of the training model. The splits were created by drawing a random sample of observations stratified by study. Receiver operating curves (ROCs) for 


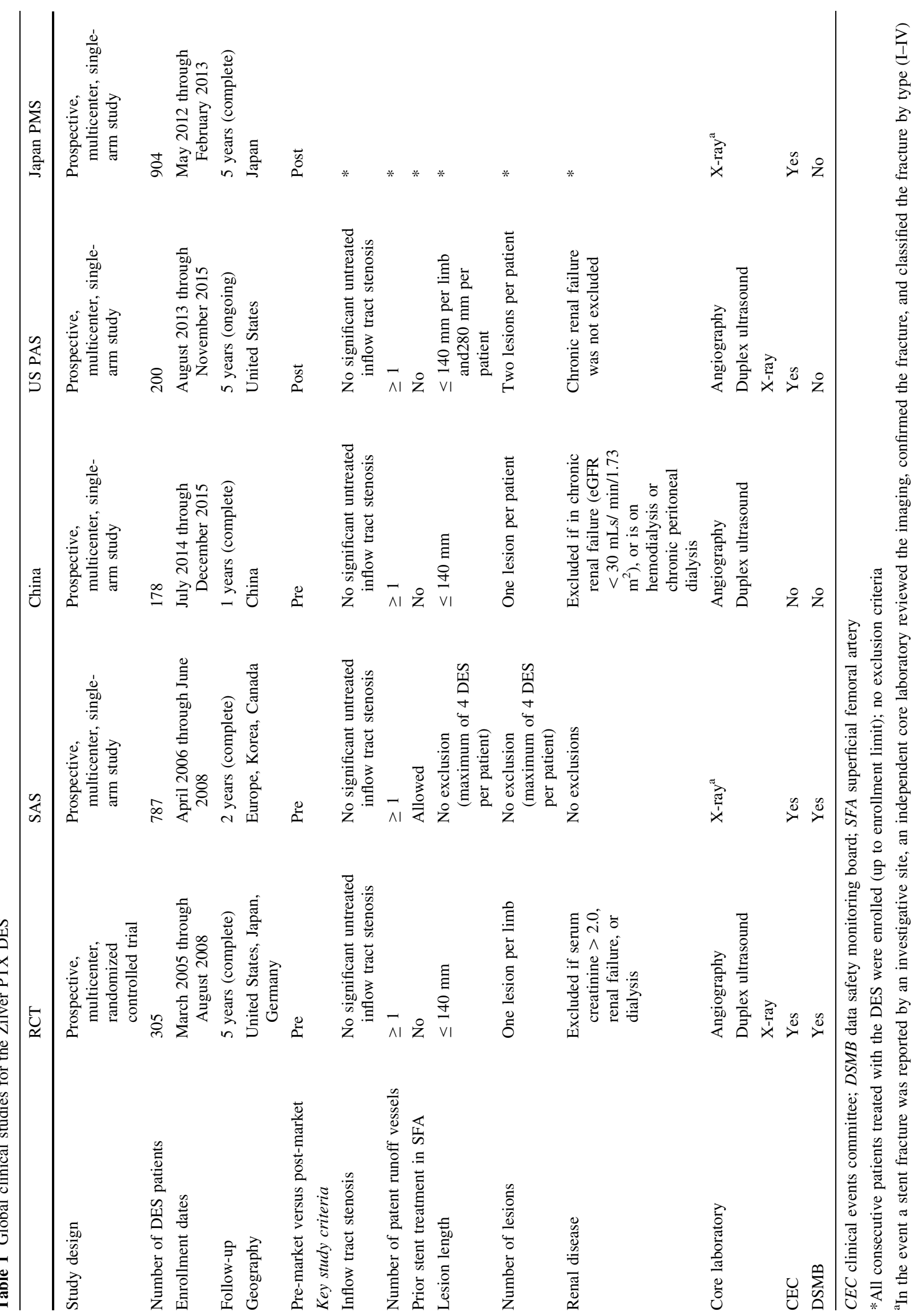


the complete data set and for training data set at 1,3 , and 5 years were generated. A concordance measure derived from the $40 \%$ test set was used. Model calibration was gauged using predicted survival plots for the training set versus the test set based on three calibration groups defined by cuts at the 27 th and $73 \mathrm{rd}$ percentiles of the distribution of the linear predictor [13].

Probability of freedom from TLR with $95 \%$ confidence intervals $(\mathrm{CI})$ was generated from the validated model at 1 , 2, 3, 4 and 5 years for combinations of patient and lesion factors composing a patient profile. This prediction model is available as an interactive web-based tool (https:// cooksfa.z13.web.core.windows.net). Analyses were conducted using SAS software, Version 9.4 of the SAS System for Windows, and Stata version 16.

Authors had access to the patient-level data used in the current analysis.

\section{Results}

Overall, there were 2227 out of 2374 (94\%) cases with complete data used for the analysis. The median follow-up time was 23.9 months (range: 0.03-60.8 months), with 61,489 months of total time at risk. For this cohort of patients, there were 1780 (79.9\%), $600(26.9 \%)$ and 443 (19.9\%) patients with follow-up through 1,3 and 5 years, respectively. The Kaplan-Meier estimates for freedom from TLR were $90.5 \%$ through 1 year and $75.2 \%$ through 5 years (Fig. 1). Table 2 shows baseline patient demographics and lesion characteristics both by individual study and overall. Hypercholesterolemia in China was notably low (18.9\%) compared to the rates reported in other studies. The rates of smoking status were variable by study. Due to study design, lesions were longer in the single-arm study as well as in the Japan post-market study. In addition, treatment of in-stent restenosis (ISR), which accounts for $60 \%$ of prior interventions, was permitted in the single-arm study as well as in the Japan post-market study. A comparison of the distribution of predictors between cases included in the model versus those omitted is shown in Supplementary Table 1 . The average RVD in the cohort of patients omitted was larger than the average RVD retained in the model; this distribution of vessel sizes was significantly different $(p=0.001)$.

Results from the univariate Cox models for factors related to reinterventions are shown in Table 3. In these analyses, diabetes, hypertension, hypercholesterolemia, RVD, calcification severity, and number of runoff vessels were not significant. To account for differences in the distribution of some patient risk factors across studies, all characteristics were included in the multivariate Cox model (Table 4). In the multivariate analysis, sex, age, Rutherford classification, lesion length, RVD, total occlusion, and
Fig. 1 Kaplan-Meier curve for freedom from TLR. Five-year freedom from TLR outcomes and $95 \%$ confidence intervals for the DES across five clinical studies. The life table is included. DES, drug-eluting stent TLR, target lesion revascularization

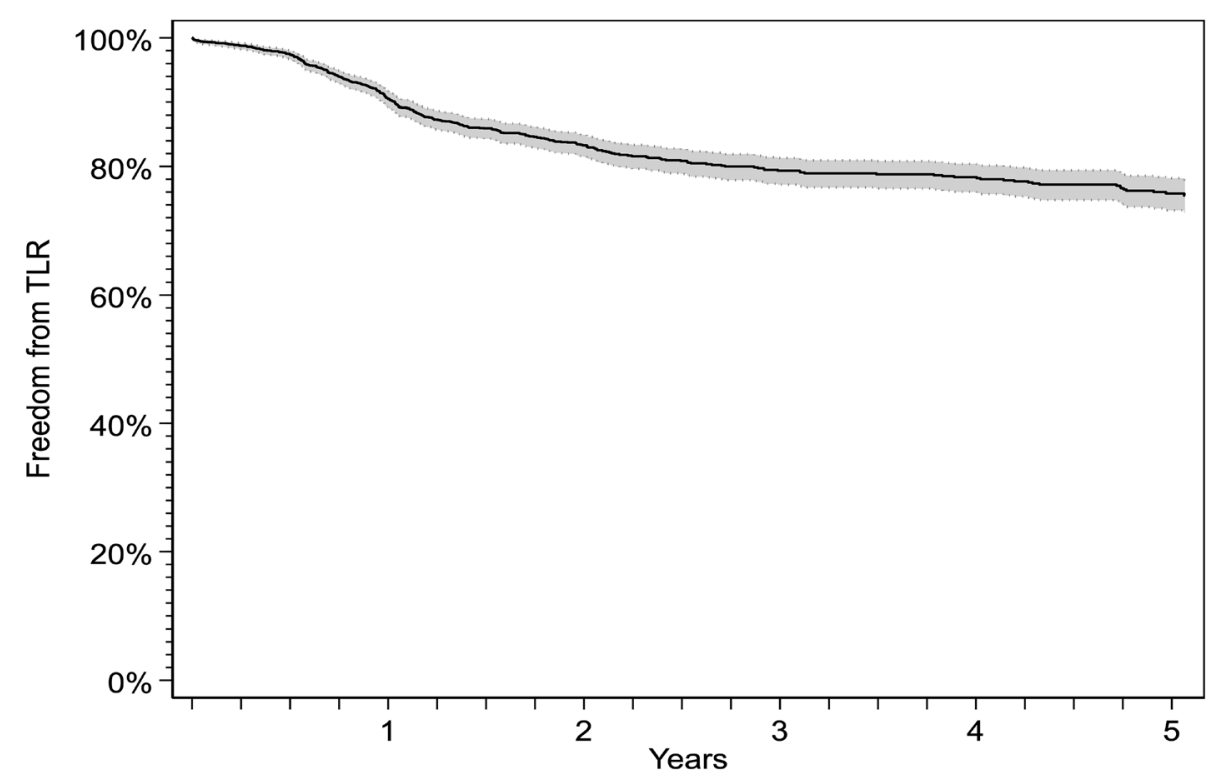

\begin{tabular}{|c|c|c|c|c|}
\hline Time (years) & $\begin{array}{c}\text { Freedom from TLR } \\
\pm \text { Standard Error }\end{array}$ & $\begin{array}{c}\text { Cumulative } \\
\text { Failed }\end{array}$ & $\begin{array}{c}\text { Cumulative } \\
\text { Censored }\end{array}$ & $\begin{array}{c}\text { Remaining } \\
\text { at Risk }\end{array}$ \\
\hline 0 & $100.0 \pm 0.0 \%$ & 0 & 0 & 2227 \\
\hline 1 & $90.5 \pm 0.6 \%$ & 200 & 247 & 1780 \\
\hline 3 & $79.4 \pm 1.0 \%$ & 356 & 1271 & 600 \\
\hline 5 & $75.2 \pm 1.3 \%$ & 378 & 1849 & 0 \\
\hline
\end{tabular}


Table 2 Baseline patient demographics and lesion characteristics

\begin{tabular}{|c|c|c|c|c|c|c|}
\hline Characteristic $^{\mathrm{a}}$ & $\begin{array}{l}\mathrm{RCT} \\
(n=301)\end{array}$ & $\begin{array}{l}\text { SAS } \\
(n=707)\end{array}$ & $\begin{array}{l}\text { China } \\
(n=175)\end{array}$ & $\begin{array}{l}\text { US PAS } \\
(n=200)\end{array}$ & $\begin{array}{l}\text { Japan PMS } \\
(n=844)\end{array}$ & $\begin{array}{l}\text { Overall } \\
(n=2227)\end{array}$ \\
\hline \multicolumn{7}{|l|}{ Sex } \\
\hline Male & $67.1 \%(202)$ & $74.4 \%(526)$ & $78.9 \%(138)$ & $63.0 \%(126)$ & $70.0 \%(591)$ & $71.1 \%(1583)$ \\
\hline Female & $32.9 \%(99)$ & $25.6 \%(181)$ & $21.1 \%(37)$ & $37.0 \%(74)$ & $30.0 \%(253)$ & $28.9 \%(644)$ \\
\hline \multicolumn{7}{|l|}{ Age } \\
\hline$<65$ & $39.2 \%(118)$ & $38.6 \%(273)$ & $40.6 \%(71)$ & $37.0 \%(74)$ & $15.6 \%(132)$ & $30.0 \%(668)$ \\
\hline $65-74$ & $33.9 \%(102)$ & $40.3 \%(285)$ & $34.9 \%(61)$ & $38.5 \%(77)$ & $37.2 \%(314)$ & $37.7 \%(839)$ \\
\hline $75-84$ & $24.6 \%(74)$ & $20.1 \%(142)$ & $21.1 \%(37)$ & $21.0 \%(42)$ & $39.3 \%(332)$ & $28.2 \%(627)$ \\
\hline$>85$ & $2.3 \%(7)$ & $1.0 \%(7)$ & $3.4 \%(6)$ & $3.5 \%(7)$ & $7.8 \%(66)$ & $4.2 \%(93)$ \\
\hline Diabetes & $48.2 \%(145)$ & $35.9 \%(254)$ & $55.4 \%(97)$ & $46.0 \%(92)$ & $59.5 \%(502)$ & $48.9 \%(1090)$ \\
\hline Hypertension & $87.7 \%(264)$ & $79.3 \%(561)$ & $76.6 \%(134)$ & $93.5 \%(187)$ & $85.4 \%(721)$ & $83.8 \%(1867)$ \\
\hline Hypercholesterolemia & $76.1 \%(229)$ & $58.1 \%(411)$ & $18.9 \%(33)$ & $86.5 \%(173)$ & $61.1 \%(516)$ & $61.2 \%(1362)$ \\
\hline Renal disease $^{\mathrm{b}}$ & $9.0 \%(27)$ & $11.3 \%(80)$ & $5.7 \%(10)$ & $13.0 \%(26)$ & $43.2 \%(365)$ & $22.8 \%(508)$ \\
\hline \multicolumn{7}{|l|}{ Smoking status } \\
\hline Never & $13.6 \%(41)$ & $17.1 \%(121)$ & $42.3 \%(74)$ & $15.5 \%(31)$ & $36.4 \%(307)$ & $25.8 \%(574)$ \\
\hline Past & $56.1 \%(169)$ & $49.4 \%(349)$ & $25.1 \%(44)$ & $43.0 \%(86)$ & $45.3 \%(382)$ & $46.3 \%(1030)$ \\
\hline Current & $30.2 \%(91)$ & $33.5 \%(237)$ & $32.6 \%(57)$ & $41.5 \%(83)$ & $18.4 \%(155)$ & $28.0 \%(623)$ \\
\hline \multicolumn{7}{|l|}{ Rutherford } \\
\hline Claudicant & $92.4 \%(278)$ & $89.5 \%(633)$ & $92.0 \%(161)$ & $86.0 \%(172)$ & $78.0 \%(658)$ & $85.4 \%(1902)$ \\
\hline CLI & $7.6 \%(23)$ & $10.5 \%(74)$ & $8.0 \%(14)$ & $14.0 \%(28)$ & $22.0 \%(186)$ & $14.6 \%(325)$ \\
\hline \multicolumn{7}{|l|}{ Lesion length } \\
\hline$<50 \mathrm{~mm}$ & $46.2 \%(139)$ & $27.6 \%(195)$ & $35.4 \%(62)$ & $27.5 \%(55)$ & $14.6 \%(123)$ & $25.8 \%(574)$ \\
\hline $50-99 \mathrm{~mm}$ & $33.9 \%(102)$ & $28.0 \%(198)$ & $36.0 \%(63)$ & $49.5 \%$ (99) & $17.2 \%(145)$ & $27.3 \%(607)$ \\
\hline $100-149 \mathrm{~mm}$ & $17.9 \%(54)$ & $15.8 \%(112)$ & $18.3 \%(32)$ & $17.0 \%(34)$ & $19.8 \%(167)$ & $17.9 \%$ (399) \\
\hline $150-199 \mathrm{~mm}$ & $1.7 \%(5)$ & $8.6 \%(61)$ & $9.1 \%(16)$ & $4.0 \%(8)$ & $9.4 \%(79)$ & $7.6 \%$ (169) \\
\hline $200-249 \mathrm{~mm}$ & $0.3 \%(1)$ & $8.9 \%(63)$ & $1.1 \%(2)$ & $1.0 \%(2)$ & $18.0 \%(152)$ & $9.9 \%(220)$ \\
\hline $250-299 \mathrm{~mm}$ & $0.0 \%(0)$ & $9.2 \%(65)$ & $0.0 \%(0)$ & $1.0 \%(2)$ & $9.7 \%(82)$ & $6.7 \%$ (149) \\
\hline$>300 \mathrm{~mm}$ & $0.0 \%(0)$ & $1.8 \%(13)$ & $0.0 \%(0)$ & $0.0 \%(0)$ & $11.4 \%(96)$ & $4.9 \%$ (109) \\
\hline \multicolumn{7}{|l|}{ RVD } \\
\hline$<5 \mathrm{~mm}$ & $42.2 \%(127)$ & $19.7 \%$ (139) & $69.1 \%(121)$ & $40.5 \%(81)$ & $19.2 \%(162)$ & $28.3 \%(630)$ \\
\hline$\geq 5 \mathrm{~mm}$ & $57.8 \%(174)$ & $80.3 \%(568)$ & $30.9 \%(54)$ & $59.5 \%$ (119) & $80.8 \%$ (682) & $71.7 \%(1597)$ \\
\hline Popliteal involvement & $5.6 \%(17)$ & $9.8 \%(69)$ & $2.3 \%(4)$ & $8.0 \%(16)$ & $7.9 \%(67)$ & $7.8 \%(173)$ \\
\hline Total occlusion & $32.6 \%(98)$ & $42.9 \%(303)$ & $50.3 \%(88)$ & $36.5 \%(73)$ & $44.4 \%(375)$ & $42.1 \%(937)$ \\
\hline \multicolumn{7}{|l|}{ Calcification } \\
\hline None & $24.9 \%(75)$ & $19.4 \%(137)$ & $24.6 \%(43)$ & $13.5 \%(27)$ & $28.1 \%(237)$ & $23.3 \%(519)$ \\
\hline Mild/moderate & $60.5 \%(182)$ & $60.0 \%(424)$ & $65.7 \%(115)$ & $69.5 \%(139)$ & $53.8 \%(454)$ & $59.0 \%(1314)$ \\
\hline Severe & $14.6 \%(44)$ & $20.7 \%(146)$ & $9.7 \%(17)$ & $17.0 \%(34)$ & $18.1 \%(153)$ & $17.7 \%(394)$ \\
\hline Prior interventions & $5.3 \%(16)$ & $25.2 \%(178)$ & $1.1 \%(2)$ & $12.5 \%(25)$ & $29.1 \%(246)$ & $21.0 \%(467)$ \\
\hline \multicolumn{7}{|c|}{ Number of runoff vessels } \\
\hline $0-1$ & $22.9 \%(69)$ & $18.1 \%(128)$ & $38.3 \%(67)$ & $22.5 \%(45)$ & $39.0 \%(329)$ & $28.6 \%(638)$ \\
\hline$>2$ & $77.1 \%(232)$ & $81.9 \%(579)$ & $61.7 \%(108)$ & $77.5 \%(155)$ & $61.0 \%(515)$ & $71.4 \%(1589)$ \\
\hline
\end{tabular}

$C L I$ critical limb ischemia; $R V D$ reference vessel diameter

${ }^{a}$ Best available data was used since a core lab was not utilized in all studies

${ }^{\mathrm{b}}$ The status for renal disease was collected as yes/no for all studies except the China study where it was collected as "chronic renal failure" $(n=0)$, "dialysis" $(n=0)$ or "other renal disease" $(n=10)$. The sum of these three measured were considered for renal disease status for the China study 
Table 3 Univariate Cox model results

\begin{tabular}{|c|c|c|c|c|c|}
\hline Characteristic $^{\mathrm{a}}$ & Hazard ratio $(95 \% \mathrm{CI})$ & $p$ value & Characteristic $^{\mathrm{a}}$ & Hazard ratio $(95 \% \mathrm{CI})$ & $p$ value \\
\hline Male & $0.699(0.567,0.861)$ & $<0.001 *$ & Male & $0.760(0.600,0.961)$ & $0.022 *$ \\
\hline \multicolumn{3}{|l|}{ Age } & \multicolumn{3}{|l|}{ Age } \\
\hline $65-74$ & $0.791(0.622,1.004)$ & $0.049^{*}$ & $65-74$ & $0.734(0.573,0.941)$ & $0.002 *$ \\
\hline $75-84$ & $0.734(0.565,0.954)$ & & $75-84$ & $0.637(0.481,0.844)$ & \\
\hline$>85$ & $0.558(0.293,1.062)$ & & $>85$ & $0.398(0.206,0.771)$ & \\
\hline Diabetes & $1.141(0.932,1.396)$ & 0.202 & Diabetes & $1.033(0.835,1.277)$ & 0.766 \\
\hline Hypertension & $0.884(0.676,1.157)$ & 0.370 & Hypertension & $0.927(0.700,1.228)$ & 0.596 \\
\hline Hypercholesterolemia & $1.089(0.880,1.347)$ & 0.434 & Hypercholesterolemia & $1.126(0.901,1.407)$ & 0.296 \\
\hline Renal disease & $1.340(1.070,1.677)$ & $0.011^{*}$ & Renal disease & $1.072(0.838,1.372)$ & 0.578 \\
\hline \multicolumn{3}{|l|}{ Smoking status } & \multicolumn{3}{|l|}{ Smoking status } \\
\hline Past & $0.726(0.570,0.925)$ & $0.024^{*}$ & Past & $0.825(0.629,1.083)$ & 0.187 \\
\hline Current & $0.918(0.704,1.196)$ & & Current & $1.020(0.753,1.383)$ & \\
\hline \multicolumn{3}{|l|}{ Rutherford } & \multicolumn{3}{|l|}{ Rutherford } \\
\hline CLI & $1.845(1.439,2.365)$ & $<0.001 *$ & CLI & $1.429(1.091,1.872)$ & $0.010^{*}$ \\
\hline \multicolumn{3}{|l|}{ Lesion length } & \multicolumn{3}{|l|}{ Lesion length } \\
\hline $50-99 \mathrm{~mm}$ & $1.607(1.122,2.300)$ & $<0.001 *$ & $50-99 \mathrm{~mm}$ & $1.443(1.003,2.074)$ & $<0.001 *$ \\
\hline $100-149 \mathrm{~mm}$ & $2.367(1.649,3.398)$ & & $100-149 \mathrm{~mm}$ & $2.066(1.425,2.994)$ & \\
\hline $150-199 \mathrm{~mm}$ & $2.416(1.551,3.763)$ & & $150-199 \mathrm{~mm}$ & $2.205(1.398,3.478)$ & \\
\hline $200-249 \mathrm{~mm}$ & $3.501(2.392,5.126)$ & & $200-249 \mathrm{~mm}$ & $2.847(1.886,4.299)$ & \\
\hline $250-299 \mathrm{~mm}$ & $3.897(2.576,5.897)$ & & $250-299 \mathrm{~mm}$ & $2.899(1.848,4.547)$ & \\
\hline$>300 \mathrm{~mm}$ & $5.130(3.381,7.784)$ & & $>300 \mathrm{~mm}$ & $3.454(2.159,5.526)$ & \\
\hline \multicolumn{3}{|l|}{ RVD } & \multicolumn{3}{|l|}{ RVD } \\
\hline$\geq 5 \mathrm{~mm}$ & $0.808(0.649,1.007)$ & 0.057 & $\geq 5 \mathrm{~mm}$ & $0.727(0.578,0.914)$ & $0.006^{*}$ \\
\hline Popliteal involvement & $1.451(1.041,2.021)$ & $0.028^{*}$ & Popliteal involvement & $1.042(0.737,1.473)$ & 0.815 \\
\hline Total occlusion & $1.882(1.536,2.305)$ & $<0.001 *$ & Total occlusion & $1.406(1.118,1.769)$ & $0.004 *$ \\
\hline \multicolumn{3}{|l|}{ Calcification } & \multicolumn{3}{|l|}{ Calcification } \\
\hline Mild/moderate & $0.953(0.749,1.213)$ & 0.752 & Mild/moderate & $0.994(0.777,1.271)$ & 0.845 \\
\hline Severe & $1.054(0.772,1.439)$ & & Severe & $1.078(0.781,1.488)$ & \\
\hline Prior interventions & $2.124(1.720,2.624)$ & $<0.001 *$ & Prior interventions & $1.815(1.443,2.282)$ & $<0.001 *$ \\
\hline \multicolumn{3}{|l|}{ Number of runoff vessels } & \multicolumn{3}{|l|}{ Number of runoff vessels } \\
\hline$\geq 2$ & $0.866(0.695,1.078)$ & 0.198 & $\geq 2$ & $0.958(0.756,1.213)$ & 0.719 \\
\hline \multirow{2}{*}{\multicolumn{3}{|c|}{$\begin{array}{l}C L I \text { critical limb ischemia; } R V D \text { reference vessel diameter } \\
* \text { Statistically significant, } p<0.05\end{array}$}} & \multirow{2}{*}{\multicolumn{3}{|c|}{$\begin{array}{l}C L I \text { critical limb ischemia; } R V D \text { re } \\
* \text { Statistically significant, } p<0.05\end{array}$}} \\
\hline & & & & & \\
\hline \multicolumn{3}{|c|}{$\begin{array}{l}{ }^{a} \text { Reference levels are-Sex: Female; Age: }<65 \text {; Diabetes: No; } \\
\text { Hypertension: No; Hypercholesterolemia: No; Renal Disease: No; } \\
\text { Smoking status: Never; Rutherford: Claudicant; Lesion length: }<50 \\
\text { mm; RVD: }<5 \mathrm{~mm} \text {; Popliteal involvement: No; Total occlusion: No; } \\
\text { Calcification: None; Prior interventions: No; Number of runoff } \\
\text { vessels: } \leq 1\end{array}$} & \multicolumn{3}{|c|}{$\begin{array}{l}{ }^{a} \text { Reference levels are-Sex: Female; Age: }<65 \text {; Diabetes: No; } \\
\text { Hypertension: No; Hypercholesterolemia: No; Renal Disease: No; } \\
\text { Smoking status: Never; Rutherford: Claudicant; Lesion length: }<50 \\
\text { mm; RVD: }<5 \text { mm; Popliteal involvement: No; Total occlusion: No; } \\
\text { Calcification: None; Prior interventions: No; Number of runoff }\end{array}$} \\
\hline
\end{tabular}

prior interventions were significant factors. The assumption of proportionality for the multivariate Cox model was met (global test, $p=0.17$ ). However, there was some evidence of non-proportionality for patients in the lesion length categories 150-199 $\mathrm{mm}$ and $>300 \mathrm{~mm}$ after adjusting for all other factors in the model.

ROCs for the complete data set and for the training data set at 1, 3, and 5 years show very similar performance
Table 4 Multivariate Cox model results

$L I$ critical limb ischemia; $R V D$ reference vessel diameter

Statistically significant, $p<0.05$ Smoking status: Never; Rutherford: Claudicant; Lesion length: $<50$ ; RVD: $<5$ mm; Popliteal involvement: No; Total occlusion: No vessels: $\leq 1$

(Fig. 2), with area under the curve (AUC) approximately 0.70 for all timepoints. The c-statistic and associated $95 \%$ CI based on the test data set was $0.70(0.66,0.74)$, suggesting adequate discrimination of the model [14]. Figure 3 shows good calibration for all three groups. Based on the validation results and the finding that the training model parameters were similar in direction and magnitude to the 

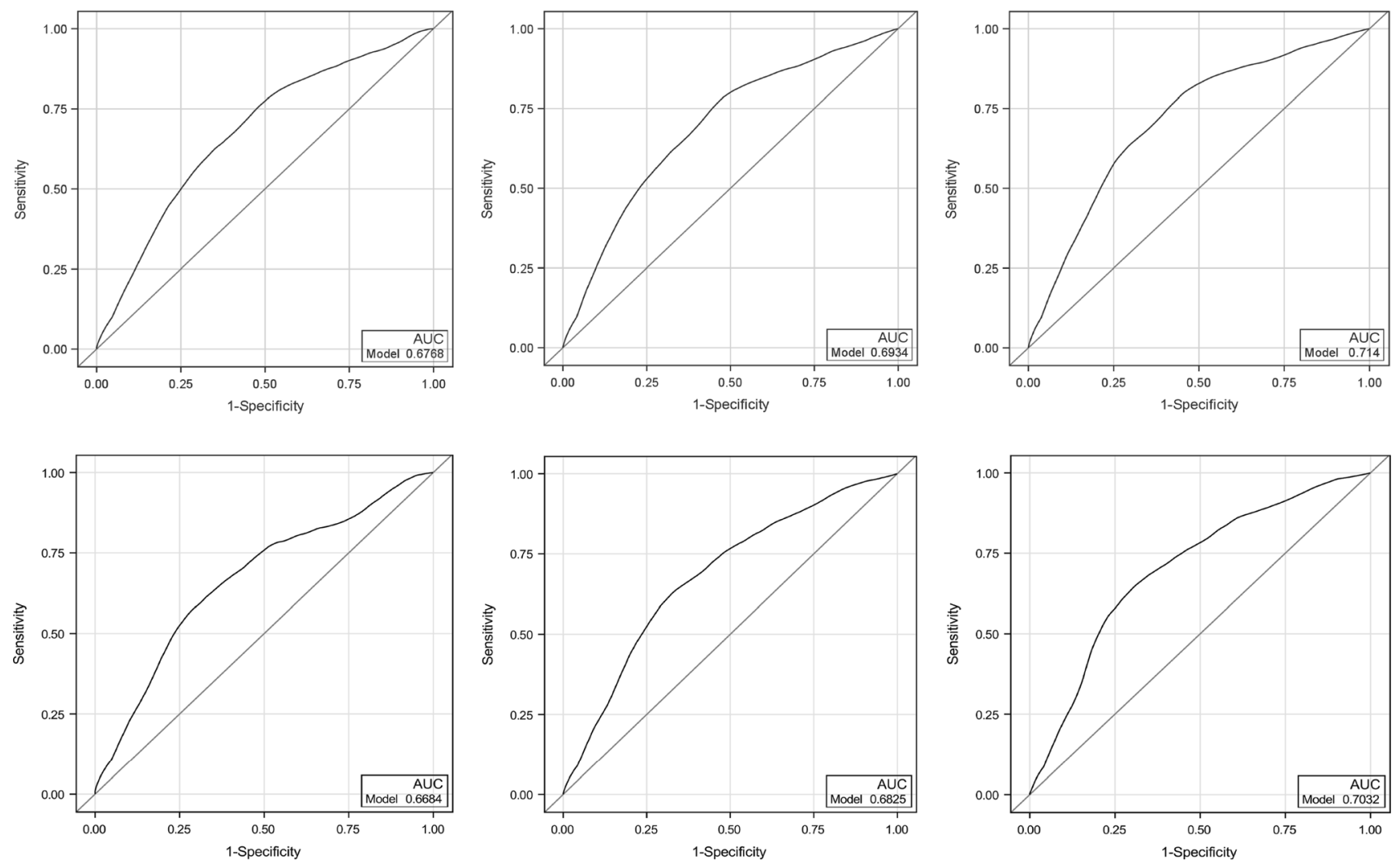

Fig. 2 Receiver operating curves for the complete data set and for the training data set. ROCs for the complete data set (top panel) and for the training data set (bottom panel) at 1,3 , and 5 years show very similar performance. ROC, receiver operating curves

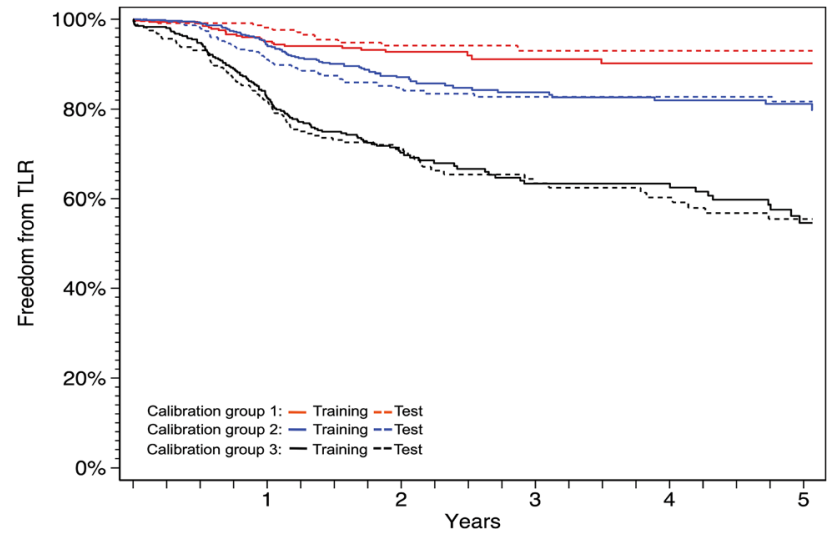

Fig. 3 Calibration curves for training data set versus test data set. Calibration groups were defined by cuts at the 27 th and $73 \mathrm{rd}$ percentiles of the distribution of the linear predictor [13]. The solid lines represent the training data set for each of the three calibration groups; the dashed lines represent the test data sets for each of the three calibration groups

parameters from the complete model, prediction results are reported from the model using the complete dataset.

Examples of three representative patient profiles are shown in Table 5. Commonly observed patient and lesion characteristics were selected in the model resulting in a range of ffTLR through 5 years for the three representative patients. The predicted freedom from TLR curves corresponding to these patient profiles is shown in Fig. 4. Through 1 year, example patient profile 1 has a predicted freedom from TLR of $97.4 \%$ (95\% CI: 96.4\%-98.5\%) and a predicted freedom from TLR of $92.8 \%$ (95\% CI: $90.1 \%$ $95.6 \%$ ) through 5 years. Example patient profile 2 has predicted freedom from TLR of $92.3 \%$ (95\% CI: 88.6\%$96.2 \%$ ) and $79.5 \%$ (95\% CI: 70.8\%-89.2\%) through 1 year and 5 years, respectively. The predicted freedom from TLR for example patient profile 3 is $86.0 \%$ (95\% CI: $79.9 \%-92.5 \%$ ) and $64.8 \%$ (95\% CI: $52.7 \%-79.7 \%$ ) through 1 year and 5 years, respectively.

\section{Discussion}

PAD affects the lower extremities and is associated with functional decline, reduced quality of life, increased depression, and increased cardiovascular morbidity and mortality [15]. Repeat treatment for PAD is also costly, inconvenient, and associated with procedural risks. As population management becomes more important, the balance between upfront procedural cost and the cost of caring for the patient in an "extended episode of care" becomes more important. The results of the data analysis 
Table 5 Example of patient profiles

Fig. 4 Predicted freedom from TLR for example patient profiles. Curves for freedom from TLR correspond to the patient characteristics outlined in Table 5. Patient profile \#1 is shown in red. Patient profile \#2 is shown in blue. Patient profile \#3 is shown in yellow. The predicted rates of freedom from TLR for each patient profile are included in the table. TLR, target lesion revascularization

\begin{tabular}{llll}
\hline Factor & Patient profile \#1 & Patient profile \#2 & Patient profile \#3 \\
\hline Sex & Male & Female & Male \\
Age & $65-74$ & $65-74$ & $75-84$ \\
Diabetes & Yes & Yes & No \\
Hypertension & Yes & Yes & Yes \\
Hypercholesterolemia & Yes & Yes & No \\
Renal disease & No & No & Yes \\
Smoking status & Past smoker & Past smoker & Past smoker \\
Rutherford classification & Claudicant & Claudicant & Claudicant \\
Lesion length & $<50 \mathrm{~mm}$ & $100-149 \mathrm{~mm}$ & $200-249$ mm \\
RVD & $\geq 5$ mm & $\geq 5$ mm & $\geq 5$ mm \\
Popliteal involvement & No & No & No \\
Occlusion & No & No & Yes \\
Calcification severity & Mild/moderate & Severe & Mild/moderate \\
Prior interventions & No & No & Yes \\
Number of runoff vessels & $2+$ & 0 or 1 & $2+$ \\
\hline
\end{tabular}

$R V D$ reference vessel diameter

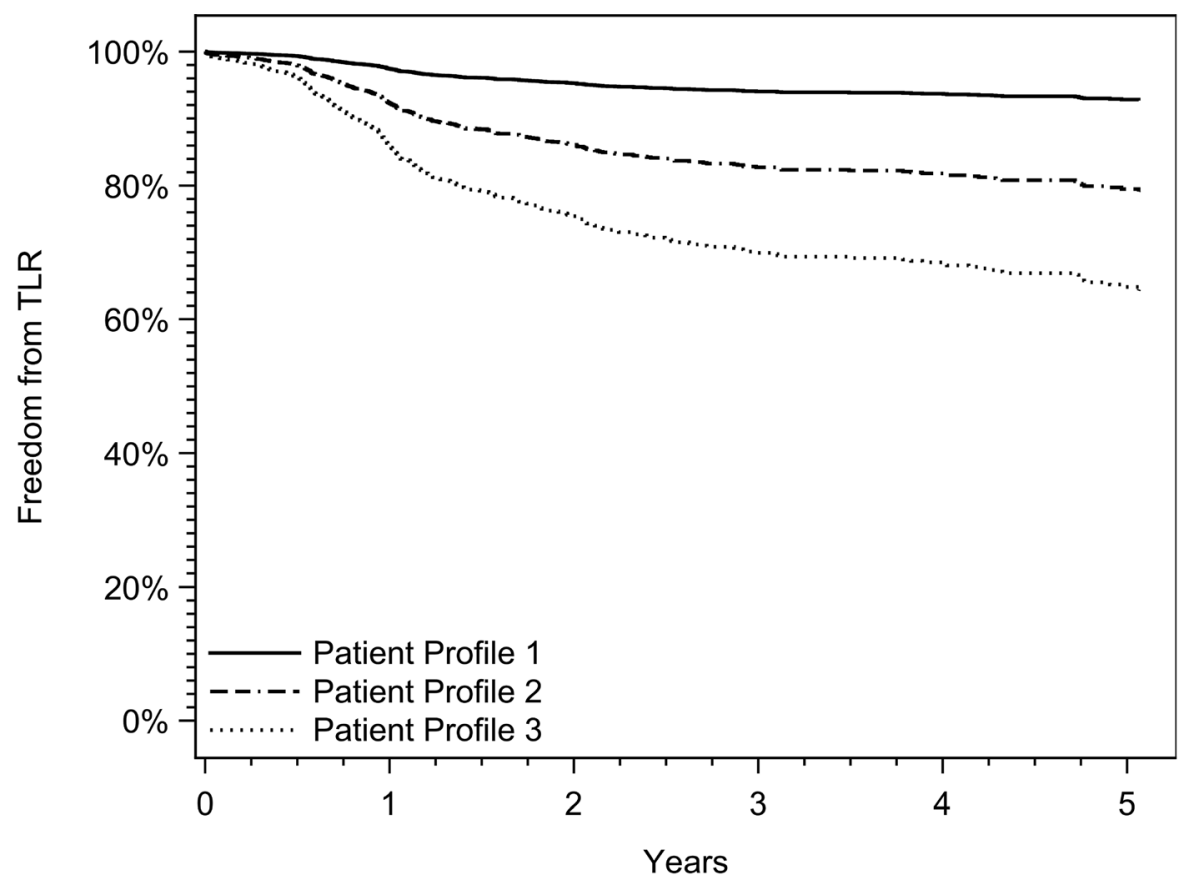

\begin{tabular}{|l|c|c|c|}
\hline Results & Patient Profile \#1 & Patient Profile \#2 & Patient Profile \#3 \\
\hline 1-year ffTLR $(95 \% \mathrm{Cl})$ & $97.4 \%$ & $92.3 \%$ & $86.0 \%$ \\
& $(96.4 \%-98.5 \%)$ & $(88.6 \%-96.2 \%)$ & $(79.9 \%-92.5 \%)$ \\
\hline 5-year ffTLR $(95 \% \mathrm{Cl})$ & $92.8 \%$ & $79.5 \%$ & $64.8 \%$ \\
& $(90.1 \%-95.6 \%)$ & $(70.8 \%-89.2 \%)$ & $(52.7 \%-79.7 \%)$ \\
\hline
\end{tabular}

identified that certain traditional risk factors commonly thought to influence reintervention rates did not have an impact, but other clinical and anatomic variables did affect the risk of TLR after DES placement.
The present study with an aggregated data analysis of 2227 patients with femoropopliteal PAD treated with the DES is the largest to date that examines the outcomes of a drug-based endovascular device according to the presence 
of patient and lesion risk factors. An analysis of the impact of these risk factors on ffTLR through 5 years of follow-up allowed creation of a prediction model. This prediction model provides a risk profile for individual patients that should markedly improve risk assessment for TLR over standard clinical variables. This model can help facilitate discussions between physicians and patients with PAD regarding expected outcomes with this DES therapy. More generally, it may contribute to improving the cost-effectiveness of care by allowing vascular specialists to optimize patient selection for DES treatment.

As anticipated, there is some variability in the frequency of individual factors between studies. In some instances, this is most likely due to differences in study enrollment criteria. For example, the Japan PMS and SAS studies enrolled longer lesions, which is reflective of the studies not excluding lesions based on length. In contrast, the other three studies limited enrollment to a maximum lesion length of $140 \mathrm{~mm}$. Smoking status, hypercholesterolemia (relatively low in the China study), and prior interventions were more frequent in the Japan PMS and SAS studies. Patients with ISR were enrolled in the Japan PMS and SAS studies in contrast to the other three studies where patients with prior stent placement were excluded. Inclusion of longer lesions and ISR strengthens the validity of the model predictions across a diverse patient population that is representative of patients commonly treated in clinical practice.

In both the univariate and multivariate analyses, the factors that were identified as significant were expected predictors of TLR including gender, age, CLI, lesion length, RVD, total occlusion, and prior interventions which includes ISR. Recent publication of 3-year results of DCB found similar risk of TLR including lesion length, RVD $\leq 4.5 \mathrm{~mm}$, ISR, bilateral disease, CLI, and hyperlipidemia [16]. Although popliteal involvement and smoking were significant in the univariate analysis, these factors were no longer significant when adjusting for other risk factors. A strong correlation between popliteal involvement and RVD is expected, and when including both in the model, RVD remained significant. This finding is consistent with observations from DCB [17]. The frequency of a RVD > $5 \mathrm{~mm}$ was more common in the Japan PMS and SAS studies. It is possible that the differences reflect regional variations in the use of intravascular ultrasound (IVUS) rather than conventional angiography to determine vessel size. Reliance on IVUS for definitive determination of RVD is a common practice and deemed a more accurate representation of true vessel size than the traditional depiction of only the lumen provided by conventional arteriography [18]. A study from Japan found a significantly higher rate of freedom from reintervention through
5 years when IVUS was used compared to non-use of IVUS [19].

There were several factors that are thought to be significant predictors of TLR; however, in this model, these factors were not significant predictors for TLR of the DES. The presence of renal disease and the number of standard tibial runoff arteries, traditionally believed to negatively impact ffTLR, did not show a significant impact in the presence of other factors included in the model. These unanticipated results are in agreement with what has been previously reported for the DES [20, 21]. While diabetes mellitus is commonly considered a risk factor for TLR following standard PTA and BMS, in this analysis diabetes did not have a significant impact, a finding previously reported in analyses of DES [22].

Patient age had a significant impact on TLR but in an unanticipated way. In this analysis, there was a decreasing risk of TLR as age increased. Although this may be counterintuitive initially, there are some possible explanations for this effect. Patients in the older age group may not be as ambulatory as younger patients. If patients are sedentary, they may not experience claudication or pain associated with exercise. In addition, older individuals in general have more medical comorbidities and may not be considered good candidates for reintervention. Although the finding was for restenosis and not TLR, analysis of the bare metal Supera stent showed an increased risk of restenosis through 3 years for patients $<75$ years old compared to patients $>75$ years old [23]. This finding is consistent with the results for age from the prediction model in which older patients had a decreased risk of TLR.

This prediction model has been adapted into an online application for clinical use by physicians (https://cooksfa. z13.web.core.windows.net). Using a patient's unique risk profile, which is based on the patient's personal clinical and lesion characteristics, the calculator predicts the risk of TLR over time. The calculator can help guide patient selection for DES placement by estimating individual risk of TLR through 5 years following SFA treatment with DES. In this study, three representative patient profiles with commonly observed patient and lesion characteristics demonstrated the functionality of this approach. In addition, this may stimulate similar modeling efforts focused on other endovascular devices with data from clinical trial experience that provides long-term follow-up. Such studies may help the field define an optimal treatment algorithm for custom management of an individual with symptomatic SFA PAD and lead to evidence-based guidance focused on maximizing the benefits and cost effectiveness of intervention.

The study and the prediction model are associated with inherent limitations. Not all of the studies have 5-year follow-up (three out of five studies with 5-year results). 
Incomplete data collection of all factors in this analysis was minimal, resulting in approximately $6 \%$ of patient cases being omitted from the analysis. The omitted cases had a higher proportion of patients with an RVD $\geq 5 \mathrm{~mm}$. These patients would be expected to have a higher ffTLR rate than those patients with smaller RVDs; therefore, the model may potentially underestimate ffTLR. Other patient characteristics were not eligible for inclusion because data for these factors were not collected in each of the five studies, including the precise type of diabetes, pulmonary dysfunction, and prior myocardial infarction. In addition, core lab analysis provides the best available data for evaluation of most anatomic factors; however, a core lab was not utilized in all studies. Accounting for all other factors in the model, the proportionality assumption was not met for two of the seven lesion length groups. Both lesion groups have considerable numbers of patients from the Japan PMS study ( $>45 \%$ of the $150-199 \mathrm{~mm}$ lesion length group and almost all of the $\geq 300 \mathrm{~mm}$ lesion length group). The authors speculate that this, along with the uniqueness of the $\geq 300 \mathrm{~mm}$ lesion length group, could contribute to the assumption not being met. Violation of this assumption suggests that a more complex relationship than what is currently presented may be relevant for this small subset of patients. Overall, because the study outcomes and prediction model are derived from a post hoc patient-level pooled analysis from five prospective clinical trials, the present results should be considered hypothesis generating.

In summary, this study evaluates a drug-based endovascular device used for management of SFA PAD and the factors that predict ffTLR for the DES. The study uses an extensive dataset to develop the first prediction model which estimates the influence of patient and lesion characteristics on ffTLR through 5 years. Based on a unique patient profile, the model provides expected patient outcomes following treatment with the Zilver PTX DES. The results from this prediction model may assist physicians to define treatment algorithms for patients as population management grows in focus.

Funding These studies were funded by Cook Medical.

\section{Compliance with Ethical Standards}

Conflict of interest MDD, AEL, HR, XJ, and GMA are paid consultants for Cook Medical. FF is a paid consultant for Cook. Medtronic, Volcano, Philips, WL Gore \& Associates. EEO'L is a paid employee of Cook Medical. MS is a paid consultant for Abbott, Endologix, and Medtronic. TZ has received honoraria from Abbott Vascular, Veryan, Biotronik, Boston Scientific Corp., Cook Medical, Gore \& Associates, Medtronic, Philips-Spectranetics, Shockwave; consulted for Boston Scientific Corp., Gore \& Associates, Medtronic, Veryan, Intact Vascular, Shockwave, Bayer, Vesper Medical; received (institution) research, clinical trial, or drug study funds from 480 biomedical, Bard Peripheral Vascular, Veryan, Biotronik, Efemoral, Cook Medical, Gore \& Associates, Medtronic, Philips, Terumo, TriReme, Shockwave, Med Alliance, Intact Vascular, B. Braun; and has common stock in QT Medical. WF, OI, and KZ report no relevant conflicts to disclose.

Ethical Approval All procedures performed in studies involving human participants were in accordance with the ethical standards of the institutional and/or national research committee and with the 1964 Helsinki Declaration and its later amendments or comparable ethical standards.

Informed Consent For the Zilver PTX randomized controlled trial (RCT), single-arm study (SAS), the China study, and the US postapproval study (US PAS), informed consent was obtained from all individual participants included in the study. For the Japan postmarket surveillance study, informed consent processes were determined by each institution's ethical committee policy to specify whether informed consent was necessary or outcome data could be abstracted while protecting patients' rights without requiring individual patient consent.

Open Access This article is licensed under a Creative Commons Attribution 4.0 International License, which permits use, sharing, adaptation, distribution and reproduction in any medium or format, as long as you give appropriate credit to the original author(s) and the source, provide a link to the Creative Commons licence, and indicate if changes were made. The images or other third party material in this article are included in the article's Creative Commons licence, unless indicated otherwise in a credit line to the material. If material is not included in the article's Creative Commons licence and your intended use is not permitted by statutory regulation or exceeds the permitted use, you will need to obtain permission directly from the copyright holder. To view a copy of this licence, visit http://creativecommons. org/licenses/by/4.0/.

\section{References}

1. Dake MD, Ansel GM, Jaff MR, Ohki T, Saxon RR, Smouse HB, et al. Paclitaxel-eluting stents show superiority to balloon angioplasty and bare metal stents in femoropopliteal disease: twelve-month Zilver PTX randomized study results. Circ Cardiovasc Interv. 2011;4(5):495-504. https://doi.org/10.1161/ circinterventions.111.962324.

2. Gray WA, Keirse K, Soga Y, Benko A, Babaev A, Yokoi Y, et al. A polymer-coated, paclitaxel-eluting stent (Eluvia) versus a polymer-free, paclitaxel-coated stent (Zilver PTX) for endovascular femoropopliteal intervention (IMPERIAL): a randomised, non-inferiority trial. Lancet. 2018;392(10157):1541-51. https:// doi.org/10.1016/s0140-6736(18)32262-1.

3. Tepe G, Laird J, Schneider P, Brodmann M, Krishnan P, Micari $A$, et al. Drug-coated balloon versus standard percutaneous transluminal angioplasty for the treatment of superficial femoral and popliteal peripheral artery disease: 12 -month results from the IN. PACT SFA randomized trial. Circulation. 2015;131(5):495-502. https://doi.org/10.1161/circulationaha.114. 011004 .

4. Rosenfield K, Jaff MR, White CJ, Rocha-Singh K, Mena-Hurtado C, Metzger DC, et al. Trial of a paclitaxel-coated balloon for femoropopliteal artery disease. $\mathrm{N}$ Engl J Med. 2015;373(2):145-53. https://doi.org/10.1056/NEJMoa1406235. 
5. Dake MD, Ansel GM, Jaff MR, Ohki T, Saxon RR, Smouse HB, et al. Durable clinical effectiveness with paclitaxel-eluting stents in the femoropopliteal artery: 5-year results of the Zilver PTX randomized trial. Circulation. 2016;133(15):1472-83. https://doi. org/10.1161/circulationaha.115.016900.

6. Schneider PA, Laird JR, Tepe G, Brodmann M, Zeller T, Scheinert D, et al. Treatment effect of drug-coated balloons is durable to 3 years in the femoropopliteal arteries: long-term results of the IN. PACT SFA randomized trial. Circ Cardiovasc Interv. 2018. https://doi.org/10.1161/circinterventions.117. 005891.

7. Konigstein M, Madhavan MV, Ben-Yehuda O, Rahim HM, Srdanovic I, Gkargkoulas F, et al. Incidence and predictors of target lesion failure in patients undergoing contemporary DES implantation-individual patient data pooled analysis from 6 randomized controlled trials. Am Heart J. 2019;213:105-11. https:// doi.org/10.1016/j.ahj.2019.03.011.

8. Dake MD, Scheinert D, Tepe G, Tessarek J, Fanelli F, Bosiers M, et al. Nitinol stents with polymer-free paclitaxel coating for lesions in the superficial femoral and popliteal arteries above the knee: twelve-month safety and effectiveness results from the Zilver PTX single-arm clinical study. J Endovasc Ther. 2011;18(5):613-23. https://doi.org/10.1583/11-3560.1.

9. Dake MD, Ansel GM, Jaff MR, Ohki T, Saxon RR, Smouse HB, et al. Sustained safety and effectiveness of paclitaxel-eluting stents for femoropopliteal lesions: 2-year follow-up from the Zilver PTX randomized and single-arm clinical studies. J Am Coll Cardiol. 2013;61(24):2417-27. https://doi.org/10.1016/j. jacc.2013.03.034.

10. Yokoi H, Ohki T, Kichikawa K, Nakamura M, Komori K, Nanto $\mathrm{S}$, et al. Zilver PTX post-market surveillance study of paclitaxeleluting stents for treating femoropopliteal artery disease in Japan: 12-month results. J Am Coll Cardiol Interv. 2016;9(3):271-7. https://doi.org/10.1016/j.jcin.2015.09.035.

11. Kichikawa K, Ichihashi S, Yokoi H, Ohki T, Nakamura M, Komori K, et al. Zilver PTX post-market surveillance study of paclitaxel-eluting stents for treating femoropopliteal artery disease in Japan: 2-year results. Cardiovasc Interv Radiol. 2019;42(3):358-64. https://doi.org/10.1007/s00270-018-2110-1.

12. Royston P, Altman DG. External validation of a cox prognostic model: principles and methods. BMC Med Res Methodol. 2013;13:33. https://doi.org/10.1186/1471-2288-13-33.

13. Cox DR. Note on grouping. J Am Stat Assoc. 1957;52(280):543-7. https://doi.org/10.1080/01621459.1957. 10501411 .

14. Hosmer DW, Lemeshow S. Applied logistic regression. 2nd ed. New York: Wiley; 2000. p. 160-164.

15. Norgren L, Hiatt WR, Dormandy JA, Nehler MR, Harris KA, Fowkes FG. Inter-society consensus for the management of peripheral arterial disease (TASC II). J Vasc Surg. 2007;45:S5-67. https://doi.org/10.1016/j.jvs.2006.12.037.
16. Torsello G, Stavroulakis K, Brodmann M, Micari A, Tepe G, Veroux $\mathrm{P}$, et al. Three-year sustained clinical efficacy of drugcoated balloon angioplasty in a real-world femoropopliteal cohort. J Endovasc Ther. 2020. https://doi.org/10.1177/ 1526602820931477.

17. Krishnan P, Tarricone A, Purushottam B, Chen S, Kapur V, Gujja $\mathrm{K}$, et al. Gender differences in the outcomes of drug-coated balloon treatment in symptomatic femoropopliteal arterial disease. Vasc Endovasc Surg. 2020;54(4):348-54. https://doi.org/10. $1177 / 1538574420911508$.

18. Shammas NW, Radaideh Q, Shammas WJ, Daher GE, Rachwan RJ, Radaideh Y. The role of precise imaging with intravascular ultrasound in coronary and peripheral interventions. Vasc Health Risk Manag. 2019;15:283-90. https://doi.org/10.2147/vhrm. S210928.

19. Iida O, Takahara M, Soga Y, Suzuki K, Hirano K, Kawasaki D, et al. Efficacy of intravascular ultrasound in femoropopliteal stenting for peripheral artery disease with TASC II class A to C lesions. J Endovasc Ther. 2014;21(4):485-92. https://doi.org/10. 1583/14-4721r.1.

20. Ogawa Y, Yokoi H, Ohki T, Kichikawa K, Nakamura M, Komori $\mathrm{K}$, et al. Impact of chronic renal failure on safety and effectiveness of paclitaxel-eluting stents for femoropopliteal artery disease: subgroup analysis from Zilver PTX post-market surveillance study in Japan. Cardiovasc Interv Radiol. 2017;40(11):1669-777. https://doi.org/10.1007/s00270-0171673-6.

21. Cipollari S, Yokoi H, Ohki T, Kichikawa K, Nakamura M, Komori K, et al. Long-term effectiveness of the Zilver PTX drugeluting stent for femoropopliteal peripheral artery disease in patients with no patent tibial runoff vessels-Results from the Zilver PTX Japan post-market surveillance study. J Vasc Interv Radiol. 2018;29(1):9-17.e11. https://doi.org/10.1016/j.jvir.2017. 08.014 .

22. Fanelli F, Di Primo M, Boatta E, Johnston K, Sapoval M. Drugeluting nitinol stent treatment of the superficial femoral artery and above-the-knee popliteal artery (the Zilver PTX single-arm clinical study): a comparison between diabetic and nondiabetic patients. Cardiovasc Interv Radiol. 2013;36(5):1232-40. https:// doi.org/10.1007/s00270-012-0543-5.

23. San Norberto EM, Flota CM, Fidalgo-Domingos L, Taylor JH, Vaquero C. Real-world results of supera stent implantation for popliteal artery atherosclerotic lesions: 3-year outcome. Ann Vasc Surg. 2020;62:397-405. https://doi.org/10.1016/j.avsg. 2019.06.038.

Publisher's Note Springer Nature remains neutral with regard to jurisdictional claims in published maps and institutional affiliations. 